\title{
What Becomes of Giant Vortices in the Abelian Higgs Model
}

\author{
Alexander A. Penin $\odot^{*}$ and Quinten Weller ${ }^{\dagger}$ \\ Department of Physics, University of Alberta, Edmonton, Alberta T6G 2J1, Canada
}

(Received 15 September 2020; accepted 16 November 2020; published 15 December 2020)

\begin{abstract}
We discuss vortex solutions of the Abelian Higgs model in the limit of large winding number $n$. We suggest a framework where a topological quantum number $n$ is associated with a ratio of dynamical scales and a systematic expansion in inverse powers of $n$ is then derived in the spirit of effective field theory. The general asymptotic form of giant vortices is obtained. For critical coupling the axially symmetric vortices become integrable in the large- $n$ limit and we present the corresponding analytic solution. The method provides simple asymptotic formulas for the vortex shape and parameters with accuracy that can be systematically improved, and can be applied to topological solitons of other models. After including the next-to-leading terms the approximation works remarkably well down to $n=1$.
\end{abstract}

DOI: 10.1103/PhysRevLett.125.251601

Vortices, stringlike solutions in theories with spontaneously broken gauge symmetry, were originally discovered in the context of superconductivity [1] and QCD confinement [2]. They play a crucial role in many physical concepts from cosmic strings [3] to mirror symmetry and dualities of supersymmetric models [4]. Giant vortices are observed experimentally in a variety of quantum condensed matter systems [5-7]. Corresponding winding numbers range from $n=4$ in mesoscopic superconductors [7], through $n=60$ in Bose-Einstein condensates of cold atoms [6] and up to $n=365$ in superfluid ${ }^{4} \mathrm{He}$ [5]. Thus, it is quite appealing to identify characteristic features and universal properties of vortices in the limit of large $n$, which is a challenging field theory problem. Though the vortex equations look deceptively simple, their analytic solution is not available. Even for critical coupling when hidden supersymmetry reduces the order of the equations [8] and even for the lowest winding number $n=1$ the solution cannot be found in a closed form [9] in contrast, for example, to the apparently more complex case of magnetic monopoles [10]. Naively, one would expect that finding analytic solutions of higher topological charge should be a bigger challenge. In general, only a few such solutions are known in gauge models (see, e.g., Refs. [11,12]). However, with increasing winding number vortices reveal some remarkable properties $[13,14]$, which indicate that in the large- $n$ limit the solution may actually become simpler. In this Letter we suggest a framework that enables a systematic expansion in inverse powers of $n$ and find the

Published by the American Physical Society under the terms of the Creative Commons Attribution 4.0 International license. Further distribution of this work must maintain attribution to the author(s) and the published article's title, journal citation, and DOI. Funded by SCOAP ${ }^{3}$. asymptotic form of the axially symmetric giant vortex solution. Moreover, for critical coupling the field equations become integrable and we present the corresponding analytic result.

Since an expansion in inverse powers of a topological charge may not be overly intuitive, let us first outline its main idea. When the winding number $n$ grows, the characteristic size of the vortex has to grow as well to accommodate the increasing magnetic flux. Assuming a roughly uniform average distribution of the flux inside the vortex we get an estimate of its radius $\sqrt{n} / e$, where $e$ is the gauge charge of the scalar field. At the same time a characteristic distance of the nonlinear interaction is $1 / e$. Thus for large $n$ we get a scale hierarchy and the expansion in the corresponding scale ratio is a standard tool of the effective field theory approach. Since we deal with the spatially extended classical solutions, it is more convenient to perform this expansion in coordinate space at the level of the equations of motion.

We consider the standard Lagrangian for the Abelian Higgs (Ginzburg-Landau) model of a scalar field with Abelian charge $e$, quartic self-coupling $\lambda$, and vacuum expectation value $\eta$ in two dimensions,

$$
L=-\frac{1}{4} F^{\mu \nu} F_{\mu \nu}+\left(D^{\mu} \phi\right)^{\dagger} D_{\mu} \phi-\frac{\lambda}{2}\left(|\phi|^{2}-\eta^{2}\right)^{2},
$$

where $D_{\mu}=\partial_{\mu}+i e A_{\mu}$. Vortices are topologically nontrivial solutions of the Euclidean equations of motion. For critical coupling $\lambda=e^{2}$ these reduce to the first-order Bogomolny equations [8]

$$
\begin{aligned}
\left(D_{1}+i D_{2}\right) \phi & =0 \\
-F_{12}+e\left(|\phi|^{2}-1\right) & =0 .
\end{aligned}
$$


We then study the axially symmetric solutions of winding number $n$, which in polar coordinates can be written as follows: $\phi(r, \theta)=f(r) e^{i n \theta}, A_{\theta}=-n a(r) / e, A_{r}=0$. It is convenient to work with the rescaled dimensionless quantities eqr $\rightarrow r, f / \eta \rightarrow f, \lambda / e^{2} \rightarrow \lambda$ so that in the new variables $e=\eta=1$ and critical coupling corresponds to $\lambda=1$. Then the Bogomolny equations in terms of the functions $a(r)$ and $f(r)$ take the following form:

$$
\begin{aligned}
& \frac{d f}{d r}-\frac{n}{r}(1-a) f=0, \\
& \frac{d a}{d r}+\frac{r}{n}\left(f^{2}-1\right)=0,
\end{aligned}
$$

with the boundary conditions $f(0)=a(0)=0$ and $f(\infty)=a(\infty)=1$. For a given winding number the solution carries $n$ quanta of magnetic flux $\Phi=$ $-\int F_{12} d^{2} \boldsymbol{r}=2 \pi n$ and the energy or string tension $T=-\int L d^{2} \boldsymbol{r}=2 \pi n \eta^{2}$.

For large $n$ the field dynamics is essentially different in three regions: the core, the boundary layer, and the tail of the vortex. Below we discuss the specifics of the dynamics and its description in each region.

The vortex core.-For small $r$ the solution of the field equations gives $f(r) \propto r^{n}$. This function is exponentially suppressed at large $n$ for all $r$ smaller than a critical value, which can be associated with the core boundary. For such $r$ the contribution of $f$ can be neglected in the equation for $a$ and we get $a(r) \approx r^{2} / r_{n}^{2}$ with $r_{n}=\sqrt{2 n}$, which in turn can be used in the equation for $f$. Thus, in the core the dynamics is described by linearized equations in the background field

$$
\begin{aligned}
\frac{d f}{d r}-\frac{n}{r}\left(1-\frac{r^{2}}{r_{n}^{2}}\right) f & =0, \\
\frac{d a}{d r}-\frac{r}{n} & =0 .
\end{aligned}
$$

Their solutions read

$$
\begin{aligned}
& f(r)=\exp \left\{\frac{n}{2}\left[\ln \left(\frac{r^{2}}{r_{n}^{2}}\right)-\frac{r^{2}}{r_{n}^{2}}+1-\frac{1}{n}\right]\right\}, \\
& a(r)=\frac{r^{2}}{r_{n}^{2}}
\end{aligned}
$$

where the form of the integration constant in the first line is determined by matching conditions explained below. For $r_{n}-r=\mathcal{O}(1)$ we have $n(1-a) / r=\mathcal{O}(1)$ and the equation for $f$ becomes independent of $n$. Hence, the approximation Eq. (4) is not applicable anymore, the nonlinear effects become crucial, and we enter the boundary layer. Note that the magnetic flux and energy density for Eq. (5) are approximately 1 and $\eta^{2}$, respectively, so that the core accommodates essentially all the vortex flux and energy and we can identify $r_{n}$ with the vortex radius.

The boundary layer.-In this region the field dynamics is ultimately nonlinear. However, it crucially simplifies for large $n$. To see this we introduce a new radial coordinate $x=r-r_{n}$ so that in the boundary layer $x=\mathcal{O}(1)$ and the expansion in $x / r_{n}$ converts into an expansion in $1 / \sqrt{n}$. In the leading order in $x$ Eq. (3) reduces to a system of $n$-independent field equations with constant coefficients

$$
\begin{aligned}
w^{\prime}+\gamma & =0, \\
\gamma^{\prime}-1+e^{2 w} & =0,
\end{aligned}
$$

where $w(x)=\ln f\left(r_{n}+x\right), \gamma(x)=n\left[a\left(r_{n}+x\right)-1\right] / r_{n}$, and prime stands for a derivative in $x$. The system can be resolved for $w$, which results in a second-order equation,

$$
w^{\prime \prime}+1-e^{2 w}=0 .
$$

This equation has a first integral $I=w^{\prime 2}-e^{2 w}+2 w$ with $I=-1$ corresponding to the boundary condition $w(\infty)=0$. Thus Eq. (6) can be solved in quadratures with the result

$$
\begin{gathered}
\int_{w_{0}}^{w(x)} \frac{\mathrm{d} w}{\left(e^{2 w}-2 w-1\right)^{1 / 2}}=x, \\
\gamma(x)=-\left[e^{2 w(x)}-2 w(x)-1\right]^{1 / 2},
\end{gathered}
$$

where $w_{0}=w(0)$ is the second integration constant. It is determined by the boundary condition $w^{\prime}(x) \sim-x$ at $x \rightarrow-\infty$, which ensures that Eq. (8) can be matched to the core solution. This gives a new transcendental constant

$$
w_{0}=-0.2997174398 \ldots
$$

which determines a unique asymptotic solution in the boundary layer. It has the Taylor expansion $w(x)=$ $\sum_{m=0}^{\infty} w_{m} x^{m}$, where $w_{1}=\left(e^{2 w_{0}}-2 w_{0}-1\right)^{1 / 2}$ and the higher order coefficients can be obtained recursively. The asymptotic behavior of the function at $x \rightarrow \infty$ reads

$$
\begin{aligned}
w(x) & \sim w_{\infty} e^{-\sqrt{2} x}, \\
w(-x) & \sim-\frac{x^{2}}{2}-\frac{1}{2}+\ldots,
\end{aligned}
$$

where $w_{\infty}=w_{0} \exp \left\{\int_{w_{0}}^{0}\left[\sqrt{2} /\left(e^{2 w}-2 w-1\right)^{1 / 2}+1 / w\right] d w\right\}$. By using Eq. (10) it is straightforward to verify that up to corrections suppressed at large $n$ the boundary layer solution Eq. (8) coincides with the core solution Eq. (5) in the matching region $1 \ll r_{n}-r \ll r_{n}$, where both 


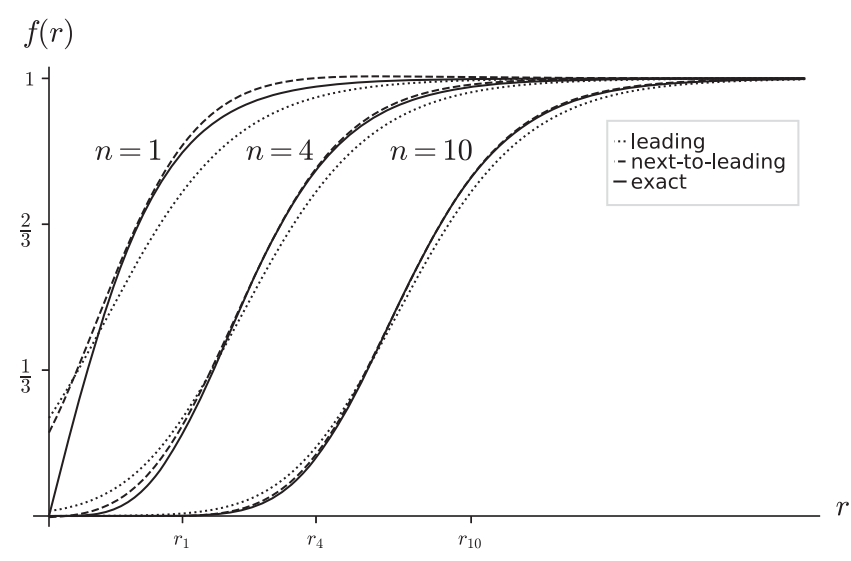

FIG. 1. The numerical solution of the exact critical vortex equations for the scalar field $f(r)$ (solid lines), the leading asymptotic solution $e^{w\left(r-r_{n}\right)}$ (dotted lines), and the next-toleading approximation (dashed lines) for different winding numbers $n$.

approximations are valid. This is a rather nontrivial result since Eq. (5) does depend on $n$.

The vortex tail.-For $\left(r-r_{n}\right) / r_{n}=\mathcal{O}(1)$ the boundary layer approximation breaks down and the coordinate dependence of the field equation coefficients should be restored. However, the deviation of the fields from the vacuum configuration is now exponentially small so the field equations linearize. The solution of the linearized theory is well known and reads

$$
\begin{aligned}
& f(r) \sim 1+\frac{\nu}{2 \pi} K_{0}(\sqrt{2} r)+\ldots, \\
& a(r) \sim 1+\frac{\mu}{2 \pi} \sqrt{2} r K_{1}(\sqrt{2} r)+\ldots
\end{aligned}
$$

where $K_{m}(z)$ is the $m$ th modified Bessel function. It describes the field of a pointlike source of scalar charge $\nu$ and magnetic dipole moment $\mu$ with $\nu=\mu$ for critical coupling. Eqs. (8) and (11) should coincide in the second matching region $1 \ll r-r_{n} \ll r_{n}$, which yields

$$
\nu=4 w_{\infty} \sqrt{\pi} e^{2 \sqrt{n}+\ln (n) / 4} .
$$

Thus vortex scalar charge and magnetic dipole moment grow exponentially with the winding number.

Calculation of the higher order terms of the expansion in $1 / \sqrt{n}$ is rather straightforward. Writing down the leading corrections to the asymptotic solutions $w$ and $\gamma$ as $\delta w / \sqrt{2 n}$ and $\delta \gamma / \sqrt{2 n}$, respectively, we get

$$
\begin{aligned}
\delta w(x) & =C w^{\prime}(x)+\int_{0}^{x} \frac{w^{\prime}(x)}{w^{\prime 2}(z)} \int_{z}^{\infty} w^{\prime 2}(y) d y d z, \\
\delta \gamma(x) & =-x w^{\prime}(x)-\delta w^{\prime}(x)
\end{aligned}
$$

where $C=\int_{-\infty}^{0}\left[z / 3+\int_{z}^{\infty} w^{\prime 2}(y) / w^{\prime 2}(z) d y\right] d z$.
Let us now consider noncritical coupling $\lambda \neq 1$. In this case the order of the field equations cannot be reduced and they read

$$
\begin{aligned}
\frac{1}{r} \frac{d}{d r}\left(r \frac{d f}{d r}\right)-\left[\lambda\left(f^{2}-1\right)+\frac{n^{2}}{r^{2}}(1-a)^{2}\right] f & =0, \\
r \frac{d}{d r}\left(\frac{1}{r} \frac{d a}{d r}\right)+2(1-a) f^{2} & =0 .
\end{aligned}
$$

Nevertheless, the general structure of the solution is quite similar to the critical case. Inside the core the contribution of the scalar potential to Eq. (14) is suppressed by $r^{2} / n^{2}$. Hence the core dynamics is not sensitive to $\lambda$ and the core solution is given by Eq. (5) up to the value of the integration constants which do depend on $\lambda$ through the matching to the nonlinear boundary layer solution. In particular the vortex size $r_{n}$ is determined by the region where the two terms in the square brackets of Eq. (14) become comparable and the core approximation breaks down, which gives the leading order result $r_{n}=\sqrt{2 n} / \lambda^{1 / 4}$. Note that the approximately constant energy density in the core is now $\lambda \eta^{2}$ so that the total vortex energy in the large- $n$ limit is $T=2 \pi \sqrt{\lambda} n \eta^{2}$. This agrees with the "wall-vortex" conjecture and numerical results for very large $n$ of Refs. [13,14]. In the tail solution, Eq. (11), the argument of $K_{0}$ gets an additional factor of $\sqrt{\lambda}$ to account for the variation of the scalar field mass, while the scalar charge and the magnetic dipole moment are not equal anymore and have different leading behavior at $n \rightarrow \infty$,

$$
|\nu| \sim e^{2 \sqrt{n} \lambda^{1 / 4}+\ldots}, \quad|\mu| \sim e^{2 \sqrt{n} / \lambda^{1 / 4}+\ldots} .
$$

More accurately, these parameters as well as the normalization of the scalar field in the core solution are determined by matching to the boundary layer solution. In the boundary layer by expanding in $x / r_{n}$ we get a system of $n$-independent equations with constant coefficients

$$
\begin{aligned}
f^{\prime \prime}-\left[\lambda\left(f^{2}-1\right)+\gamma^{2}\right] f & =0, \\
\gamma^{\prime \prime}-2 \gamma f^{2} & =0,
\end{aligned}
$$

with the boundary condition $\gamma(x) \sim \sqrt{\lambda} x$ at $x \rightarrow-\infty$. For $\lambda=1$ the proper solution is given by Eq. (8) and for any given $\lambda \neq 1$ it can be found numerically.

Finally, we briefly discuss a simpler but quite interesting case of the vortices in Bose-Einstein condensate of a neutral scalar field. The corresponding vortex equation is obtained from the first line of Eq. (14) by setting $a=0$ and $\lambda=1$ (see, e.g., Ref. [15]). Now the dynamics in the core of radius $r_{n}=n$ is described by a linear differential equation, while in the tail region $r_{n}+\delta \lesssim r$ with $\delta \propto n^{1 / 3}$ the derivative term is suppressed and the field equation becomes algebraic at $n \rightarrow \infty$. In contrast to the charged 


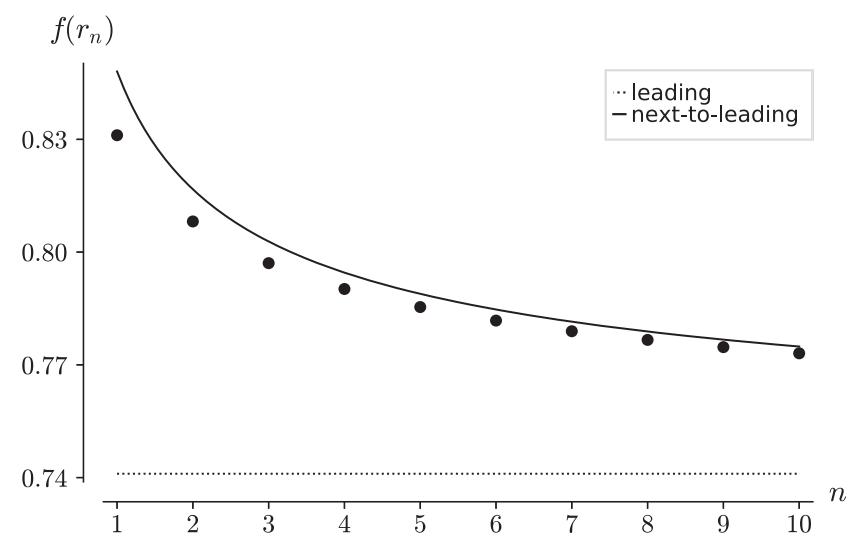

FIG. 2. The values $f\left(r_{n}\right)$ obtained from the numerical solution of the exact critical vortex equations (black dots), the asymptotic value $f\left(r_{n}\right)=e^{w_{0}}$ (dotted line), and the next-to-leading approximation (solid line) as functions of the winding number $n$.

case the boundary layer does not form and the core and tail solutions can be polynomially matched over the interval $r_{n} \lesssim r \lesssim r_{n}+\delta$. This yields the asymptotic solution

$$
f(r)= \begin{cases}C^{\prime} J_{n}(r), & r \leq r_{n}, \\ \sqrt{\delta / 2 n}\left[1+\left(r-r_{n}\right) / \delta\right], & r_{n}<r<r_{n}+\delta, \\ \sqrt{1-n^{2} / r^{2}}, & r_{n}+\delta \leq r,\end{cases}
$$

where $J_{n}(r)$ is the $n$th Bessel function, $C^{\prime}=$ $\left(3^{1 / 4} \pi^{1 / 2} / 2^{1 / 2}\right), \delta=\left(2^{2 / 3} \pi n^{1 / 3} /\left[3^{5 / 6} \Gamma^{2}(2 / 3)\right]\right)$, and $\Gamma(z)$ is the Euler gamma function. The vortex energy now is $T=\pi \eta^{2} n^{2}$, where a half of the contribution comes from the vortex tail. Curiously, for $\eta=1$ it is given by the area of a circle of radius $n$ while the critical vortex energy is equal to the corresponding circumference. The corrections to Eq. (17) are given by a series in $1 / n^{1 / 3}$ and will be published elsewhere.

The results of numerical analysis of the large- $n$ approximation are presented in Figs. 1-5. In Fig. 1 the leading asymptotic result $e^{w\left(r-r_{n}\right)}$ and the next-to-leading approximation which incorporates the $\mathcal{O}(1 / \sqrt{n})$ terms are plotted against numerical solutions of the exact field equations for $f(r)$ with $\lambda=1, n=1,4,10$. In Figs. 2 and 3 the exact numerical values of $f\left(r_{n}\right)$ and $\nu$, the natural characteristics of the vortex solution, are plotted against the asymptotic leading and next-to-leading results for $\lambda=1, n \leq 10$. The expansion reveals an impressive convergence and the nextto-leading approximation works reasonably well even for $n=1$. For completeness we present the result for the asymptotic profile of the boundary layer solution for the scalar field with $\lambda=1 / 2,1,2$ in Fig. 4. The numerical results for a neutral scalar field vortex with $n=10$ are given in Fig. 5.

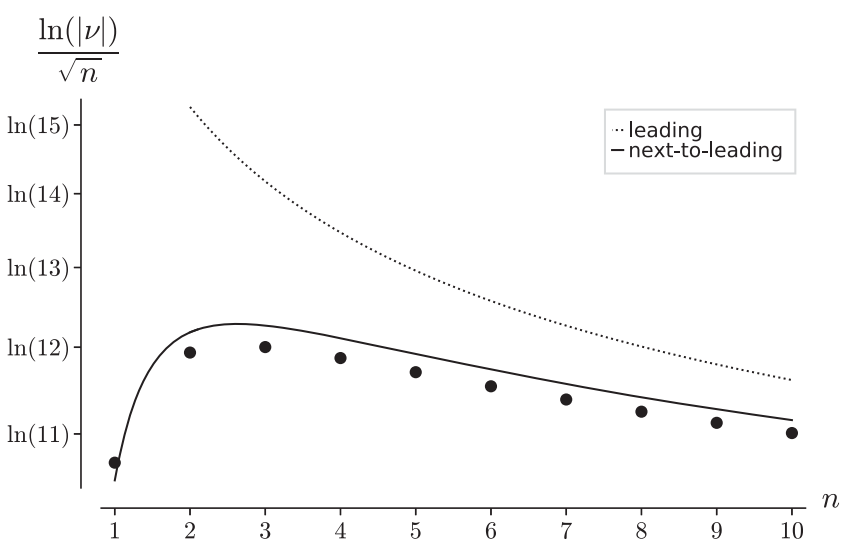

FIG. 3. Scalar charge or magnetic dipole moment $\nu$ of a critical vortex obtained from the numerical solution of the exact vortex equations (black dots), the asymptotic result Eq. (12) (dotted line), and the next-to-leading approximation (solid line) as functions of the winding number $n$.

To summarize, we have elaborated a method of expansion in inverse powers of a topological quantum number. The method is quite general and can be applied to the study of topological solitons in a theory where the corresponding quantum number can be associated with a ratio of dynamical scales, e.g., to the multimonopole solutions in the YangMills Higgs model, where only the case of vanishing scalar potential has been solved so far. When applied to axially symmetric vortices with large winding number $n$ the expansion is in powers of $1 / n^{1 / \alpha}$ with $\alpha=2$ for the charged and $\alpha=3$ for the neutral scalar field. In the large- $n$ limit the complex nonlinear vortex dynamics unravels. In particular, the field equations become integrable for critical coupling and reduce to an algebraic one for a neutral Bose-Einstein condensate. This yields simple asymptotic formulas for the shape and parameters capturing the main features of the giant vortices. The accuracy of the asymptotic result can be systematically improved and already after including the

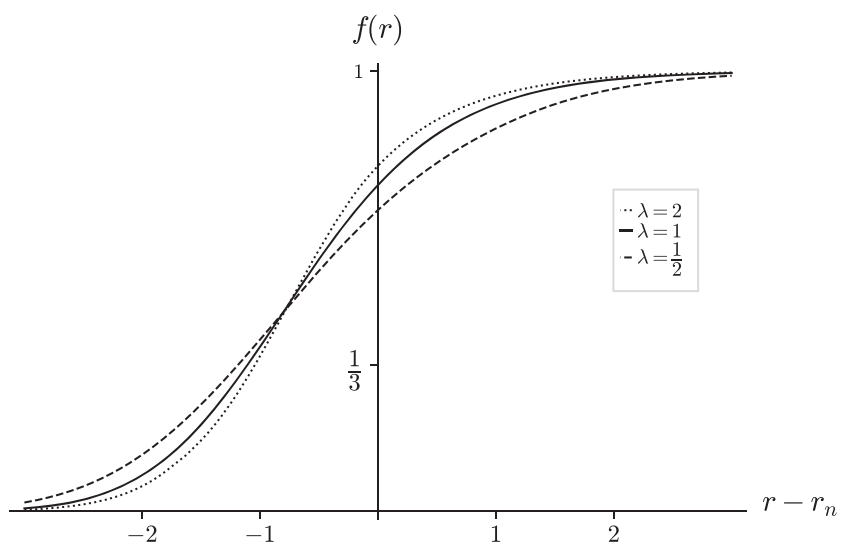

FIG. 4. The asymptotic profiles of the scalar field $f(r)$ obtained by numerical solution of the effective vortex equations Eq. (16) for different values of scalar self-coupling as functions of $r-r_{n}$. 


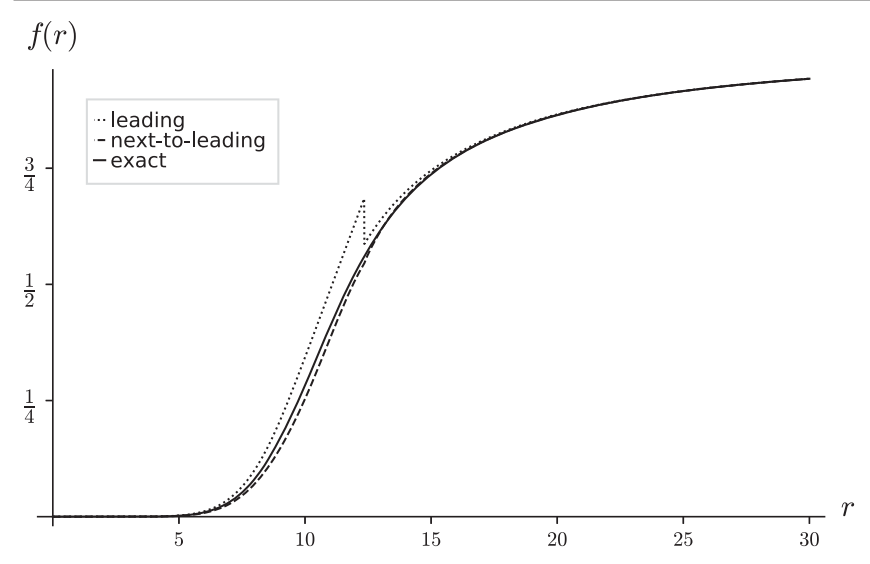

FIG. 5. The numerical solution of the exact vortex equations for a neutral scalar field $f(r)$ (solid line), the leading asymptotic solution Eq. (17) (dotted line), and the next-to-leading approximation (dashed line) for $n=10$.

leading corrections the approximation works remarkably well all the way down to very low $n$.

A. P. is grateful to Joseph Maciejko for useful communications. The work of A.P. was supported in part by NSERC and the Perimeter Institute for Theoretical Physics. The work of Q. W. was supported through the NSERC USRA program.

\section{*penin@ualberta.ca} †weller@ualberta.ca

[1] A. A. Abrikosov, Zh. Eksp. Teor. Fiz. 32, 1442 (1957) [Sov. Phys. JETP 5, 1174 (1957)].

[2] H. B. Nielsen and P. Olesen, Nucl. Phys. B61, 45 (1973).

[3] M. B. Hindmarsh and T. W. B. Kibble, Rep. Prog. Phys. 58, 477 (1995).

[4] D. Tong, arXiv:hep-th/0509216.

[5] P. L. Marston and W. M. Fairbank, Phys. Rev. Lett. 39, 1208 (1977).

[6] P. Engels, I. Coddington, P. C. Haljan, V. Schweikhard, and E. A. Cornell, Phys. Rev. Lett. 90, 170405 (2003).

[7] T. Cren, L. Serrier-Garcia, F. Debontridder, and D. Roditchev, Phys. Rev. Lett. 107, 097202 (2011).

[8] E. B. Bogomolny, Yad. Fiz. 24, 861 (1976) [Sov. J. Nucl. Phys. 24, 449 (1976)].

[9] H. J. de Vega and F. A. Schaposnik, Phys. Rev. D 14, 1100 (1976).

[10] M. K. Prasad and C. M. Sommerfield, Phys. Rev. Lett. 35, 760 (1975).

[11] E. Witten, Phys. Rev. Lett. 38, 121 (1977).

[12] M. K. Prasad and P. Rossi, Phys. Rev. Lett. 46, 806 (1981).

[13] S. Bolognesi, Nucl. Phys. B730, 127 (2005).

[14] S. Bolognesi and S. B. Gudnason, Nucl. Phys. B741, 1 (2006).

[15] E. M. Lifshitz and L.P. Pitaevskii, Statistical Physics, Part 2: Theory of the Condensed State, Vol. 9, 1st ed. (Butterworth-Heinemann, Oxford, 1980). 